\section{Supraversión de la mirada en intoxicación mortal por bupropión}

\author{
LUIS CARTIER R., CRISTIAN ROMERO O., GERARDO PÉREZ P.
}

\section{Sustained upgaze in a patient who ingested high doses of bupropion. Report of one case}

Upgaze or sustained elevation of the eyes, is an alteration of ocular motility initially described in hypoxic coma. We report a 65-year-old woman admitted with hypotension and alteration of sensorium due to the ingestion of $9.5 \mathrm{~g}$ of Bupropion. She presented two seizures of short duration, without epileptic activity on the EEG. She had a persistent asynchronous myoclonus in extremities, tachycardia and prolonged Q-t. She suffered a cardiac arrest caused by asystole, which recovered quickly in five minutes. At that moment, upgaze appeared, associated with a persistent ocular opening, which persisted for days, but finally disappeared, without remission of coma. A magnetic resonance imaging done at the eighth day, showed hyperintensity of the oval center and corpus callosum which disappeared in a new imaging study done 30 days later, where images of hypoxia in the basal nuclei and cortex appeared. The patient died forty seven days after admission. Up-gaze is an ominous oculomotor alteration linked to an important but incomplete damage in the cerebral cortex, a condition that perverts some sequences of the ocular opening, reversing the Bell phenomenon and producing eyelid retraction.

(Rev Med Chile 2018; 146: 665-669)

Key words: Bupropion; Coma; Hypoxia; Leukoencephalopathies.
Departamento de Ciencias

Neurológicas Oriente, Facultad de

Medicina Universidad de Chile.

Servicio de Neurología, Hospital

del Salvador, Santiago, Chile.

Trabajo no recibió

financiamiento.

Los autores declaran no tener

conflictos de interés.

Recibido el 30 de septiembre de 2017, aceptado el 25 de mayo de 2018.

Correspondencia a:

Dr. Luis Cartier Rovirosa

Fono: 66489936

Icartier@med.uchile.cl
$\mathrm{L}$ a supraversión de la mirada es una elevación anormal de los ojos al abrirlos, haciendo que automáticamente la mirada se dirija hacia arriba, alterando su natural postura central, condición que además se asocia a persistente apertura ocular y escaso pestañeo. La supraversión de la mirada es una alteración que se ha comunicado en relación a encefalopatías hipóxicas, generalmente producto de un paro cardiorrespiratorio o hipotensiones prolongadas y cuya neuropatología ha revelado daño neuronal de la corteza cerebral y cerebelosa con preservación del tronco cerebral ${ }^{1,2}$.

Las intoxicaciones agudas con dosis altas de bupropión pueden llevar al coma. El bupropión es un antidepresivo que actúa inhibiendo la recaptación de dopamina, norepinefrina, serotonina y también antagoniza receptores nicotínicos de la acetilcolina. La dosis terapéutica fluctúa entre $150 \mathrm{mg}$ y $450 \mathrm{mg} /$ día y la vida media del medicamento va de 10 a $20 \mathrm{~h}$. Es degradado por enzimas hepáticas, que dejan metabolitos activos como hidroxibupropión, treo-hidroxibupropión y eritrohidrobupropión que pueden persistir más tiempo ${ }^{3}$.

Aún en dosis terapéuticas el bupropión puede producir síntomas adversos, como insomnio, déficit atencional, inquietud psicomotora, temblor y convulsiones, eventualmente alucinaciones, también taquicardia, hipertensión arterial y trastorno gastrointestinales ${ }^{4}$. La intoxicación por sobreingestión del bupropión acentúa los síntomas adversos, pudiendo desencadenar estatus epilépticos, llegar al coma y ser fatal ${ }^{5}$. Un tercio de los pacientes intoxicados con bupropión desarrollan crisis epilépticas, lo que puede aumentar a 100\% de acuerdo con la dosis ${ }^{6}$. Son de importancia y de- 
ben ser previstos los efectos cardiotóxicos, donde destaca la taquicardia y el alargamiento de Q- $t$ en el electrocardiograma, que podrían conducir a paro cardíaco ${ }^{7}$.

Presentamos una paciente que desarrolló una supraversión de la mirada en relación a una grave hipoxia por complicaciones cardiotóxicas producidas por sobredosis de bupropión.

\section{Caso clínico}

Se presenta el caso de una mujer de 62 años en tratamiento por trastorno de personalidad y del ánimo, con el antecedente de dos intentos suicidas. En febrero de 2017 ingirió bupropión en dosis de 9,5 g, clorpromazina $1,5 \mathrm{~g}$ y dos copas de vino. Los familiares la encontraron alterada y la llevaron al Hospital del Salvador, donde ingresó comprometida de conciencia en Glasgow 10, presión arterial 90/60, taquicárdica $115 \mathrm{lpm}$, electrocardiograma con segmento Q-t alargado (550 ms) y ácido láctico elevado $(32,6 \mathrm{mg} / \mathrm{dl})$. Dentro de las primeras $24 \mathrm{~h}$ de su ingreso presentó dos breves episodios convulsivos, de 30 seg y de menos de un minuto. El electroencefalograma (EEG) mostró actividad delta-theta de bajo voltaje, sin signos de epilepsia. La tomografía de cerebro (TAC) sin contraste fue normal. La paciente tenía escaza reactividad y se mantuvo alterada de conciencia, con mioclonías generalizadas y movimientos involuntarios de las extremidades; tono muscular aumentado, reflejos osteotendíneos (ROT) policinéticos y reflejos plantares flexores. En la mañana del tercer día sufrió paro cardiorrespiratorio por asistolia, recibió reanimación inmediata, adrenalina, intubación, luego de 5 min logró actividad cardiaca espontánea.

Al día siguiente habían desaparecido las mioclonías, estaba inconsciente y mantenía una persistente apertura palpebral con escaso pestañeo y con una sobre-elevación anormal de los ojos, como mirando hacia atrás. Las pupilas estaban iguales y reactivas a la luz, con reflejos oculocefálicos conservados. Sus extremidades se mostraban hipotónicas y tenía los ROT exaltados con Babinski bilateral. Los estímulos nociceptivos desencadenaban respuestas extensoras de las cuatro extremidades y un simultáneo cierre palpebral con descenso de los globos oculares, que en la reapertura palpebral volvían a elevarse simulando un rebote ocular. El fenómeno se repetía con cualquier estímulo. La
RM de cerebro realizada al octavo día, mostró en la difusión imágenes hiperintensas de la sustancia blanca del centro oval y del cuerpo calloso y en secuencia FLAIR áreas menores hiperintensas en ganglios basales y corteza, compatibles con una encefalopatía hipóxico-isquémica (Figura 1).

La supraversión de la mirada se mantuvo al menos diez días, luego la mirada se hizo central, mientras la paciente seguía en coma y sin variaciones después de un mes. Las pupilas se mantenían iguales con respuesta a la luz y reflejos oculocefálicos conservados, los ROT estaban vivos con reflejos plantares extensores. Los estímulos nociseptivos seguían generando respuestas extensoras. El EEG estaba prácticamente plano. La RM de control mostró una definida atrofia cerebral, con hiperintensidades en los núcleos de la base y en distintas áreas de la corteza cerebral y cerebelosa. Había desaparecido la hiperintensidad de la substancia blanca (Figura 2). La paciente falleció a los 47 días.

\section{Comentario}

La ingestión de más de 60 comprimidos de bupropión, condujeron a la alteración de conciencia, la aparición de crisis convulsivas, así como de un persistente aumento de la frecuencia cardiaca sobre $110 \mathrm{l} / \mathrm{m}$ y una hipotensión bajo $90 \mathrm{~mm}$, confirmando lo que se ha descrito en estas intoxicaciones ${ }^{4}$. De modo que la mínima ingesta de alcohol y la baja dosis de clorpromazina ingerida no pueden explicar el cuadro. El perfil clínico de su ingreso parecía sugerir una crisis serotoninérgica, expresada por el estado confusional, la taquicardia, el aumento del tono muscular, la hiperreflexia y actividad mioclónica ${ }^{8}$. El cuadro se agravó después del breve paro cardíaco al acentuar la hipoxia de la hipotensión prolongada, al efecto del bupropión sobre el sistema nervioso central. En las intoxicaciones agudas por bupropión la hipoxia ha sido una complicación excepcional ${ }^{9}$.

La supraversión de la mirada que desarrolló esta paciente en el contexto de su coma neurológico, fue descrita en 1981 por Keane en pacientes con daño hipóxico del SNC. Keane estudió 13 pacientes que mostraban una anormal y persistente elevación de los ojos sobre la línea media durante el coma. Esta alteración de la mirada duró varios días, como en nuestra paciente. Además, describió 
la aparición de nistagmos vertical hacia abajo en 6 de sus 13 casos $^{1}$. Este curioso trastorno de la mirada fue interpretado como una liberación de reflejos vestibulares expresados en concordancia con la hipoxia del flóculo y la indemnidad de la protuberancia ${ }^{2}$.
Creemos que la supraversión de la mirada es una alteración mucho más compleja que la pérdida del control de los reflejos vestibulares, como consecuencia de una anoxia cerebelosa. La supraversión de la mirada parece en lo esencial una perturbación del fenómeno de Bell, donde

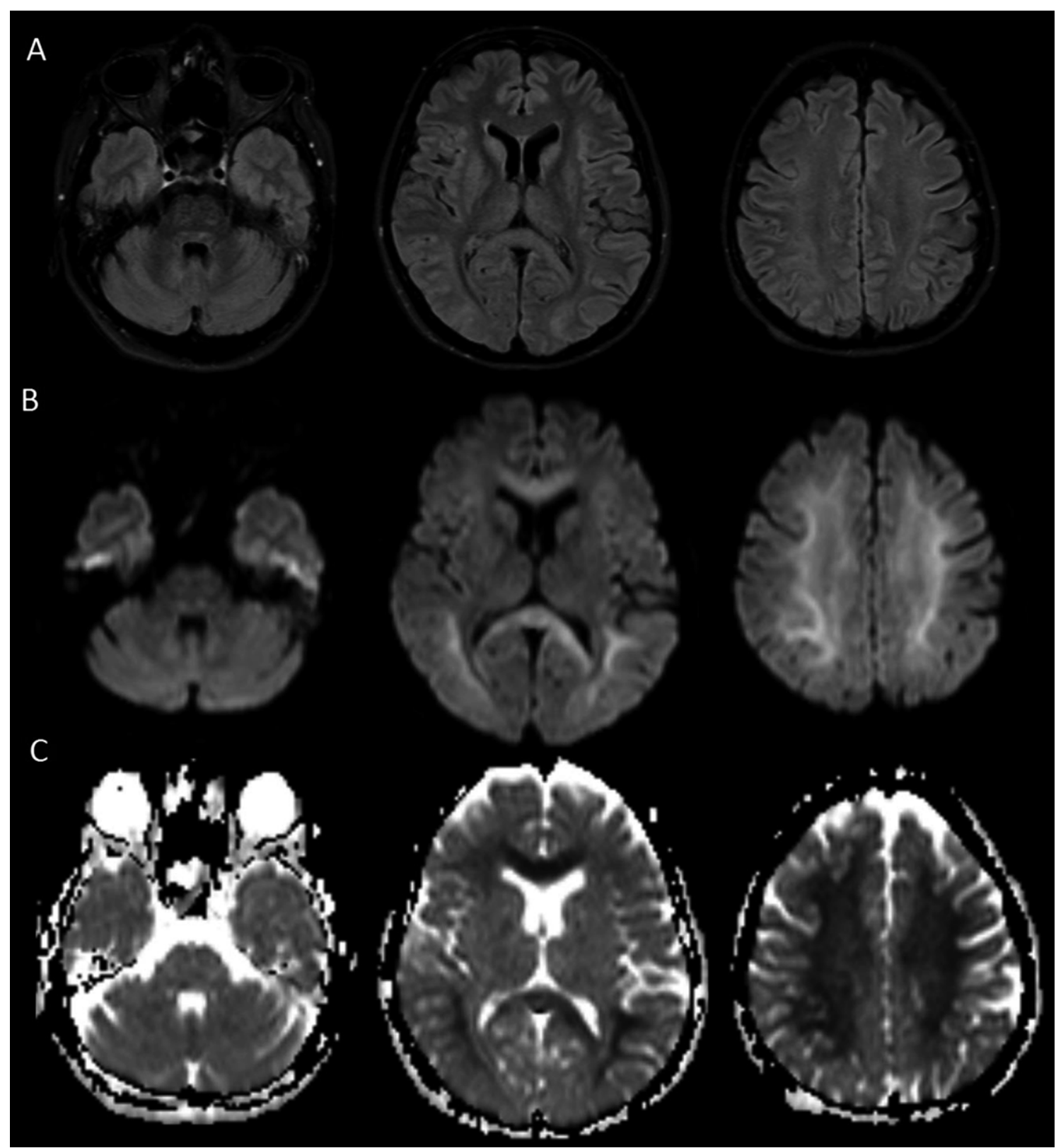

Figura 1. Resonancia magnética de cerebro al octavo día. A) secuencia FLAIR: Aumento de señal en la corteza cerebral y en los ganglios basales, menos significativa en la corteza cerebelosa. B) secuencia DWI: Importante hiperintensidad de la substancia blanca en el cuerpo calloso y en el centro oval, con una apariencia leucoencefalopática. C) secuencia ADC: Hipointensidad de la substancia blanca en las mismas estructuras. 
lo normal es que los ojos se eleven al cerrarlos, en estos pacientes los ojos se elevan al abrir los párpados, además la elevación ocular se asocia a una persistente apertura palpebral, configurando un trastorno oculomotor cuya fisiopatogenia parece depender de áreas prefrontales ${ }^{10}$. La persistente apertura palpebral es asimilable a la "retracción palpebral", alteración observada en la enfermedad de Parkinson y en la enfermedad de Creutzfeldt-Jakob, de definido origen cortical ${ }^{11}$. Además, en

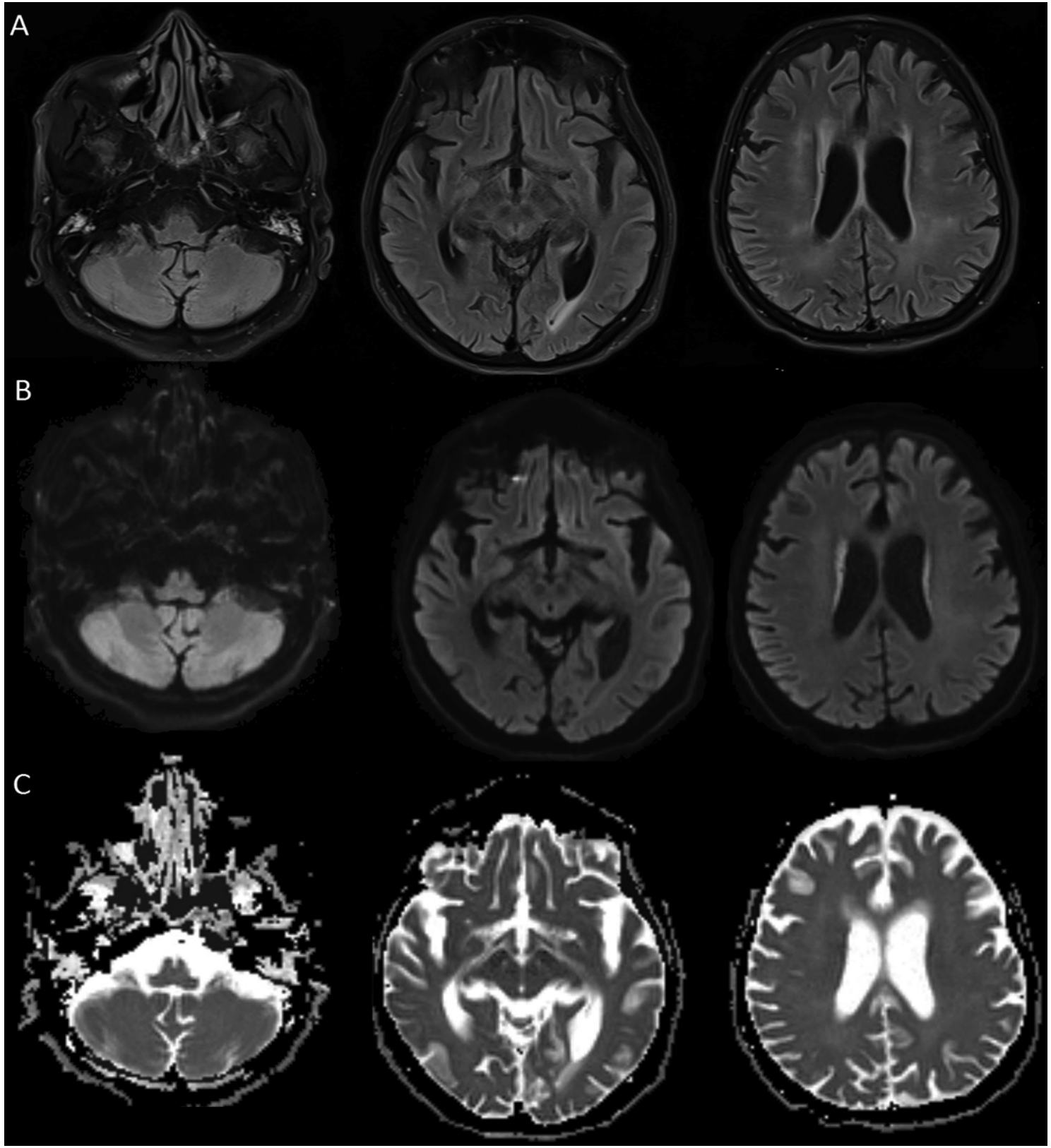

Figura 2. Resonancia magnética de cerebro treinta días después. A) secuencia FLAIR: Atrofia cerebral, con significativo aumento de la señal en los núcleos de la base, en distintas áreas de la corteza cerebral y cerebelosa, también periventricular. B) secuencia DWI: Desaparición del aumento de señal en la substancia blanca, observándose un definido adelgazamiento del cuerpo calloso. C) secuencia ADC: La substancia blanca se ve conservada. 
esta paciente los estímulos nociseptivos desencadenaban crisis extensoras de las extremidades y un simultáneo cierre de los párpados con descenso de los globos oculares y un fenómeno de rebote con elevación ocular a la apertura palpebral.

La supraversión de la mirada traduce una grave disfunción cortical como expresión de un amplio aunque incompleto daño de la corteza cerebral. Sabemos que la corteza es asiento de variados y esenciales esquemas motores como la apertura y cierre voluntario de los ojos ${ }^{12}$ y resulta difícil comprender esta compleja alteración como una liberación de reflejos vestibulares. Por otra parte, el carácter ominoso de la supraversión de la mirada y su especificidad, hace comparable este fenómeno a la clásica inversión del reflejo plantar que lo convierte en signo de Babinski.

También es de interés resaltar en esta paciente, la precoz injuria de la substancia blanca expresada por la hiperintensidad simétrica del centro oval, del cuerpo calloso y de la cápsula interna (Figura 1) imágenes que parecían denunciar un compromiso primario de la substancia blanca. Sin embargo, esas imágenes, sorprendentes por lo excepcionales, habían sido comunicadas en una serie de siete pacientes, entre quienes esta alteración había aparecido en la primera semana de sendos comas hipóxicos, cuyas causas primarias eran: sobredosis de medicamentos (3/7), paro cardiaco (2/7), taquicardia paroxística (1/7) e hipotensión $(1 / 7)^{13}$. Este modo de alteración primaria de la substancia blanca ha llamado la atención porque es inhabitual en la hipoxia y difiere de la clásica leucoencefalopatía hipóxica de Ginsberg, que es tardía y multifocal ${ }^{14}$. Por lo excepcional del fenómeno nos pareció que adquiría relevancia la convergencia del antecedente tóxico suicida, que se observó en la mitad de los enfermos comunicados, incluyendo nuestra paciente. Esa pre-condición pudo contribuir a una precoz alteración en la permeabilidad de la barrera hematoencefálica, que logró los cambios transitorios de la RM. De manera clásica, la falta de oxígeno afecta primordialmente las neuronas, condición que se traduce en imágenes que comprometen especialmente dichas estructuras, semejantes a la figura 2, última RM de la enferma ${ }^{15}$. No cabe duda que para desarrollar esa precoz hiperintensidad de la substancia blanca en la RM se requeriría de factores adicionales, como la intoxicación con bupropión.

\section{Referencias}

1. Keane JR. Sustained Upgaze in Coma. Ann Neurol 1981; 9: 409-12.

2. Nakada T, Kwee IL, Lee H. Sustained up gaze in coma. J Clin Neuro-ophthalmol 1984; 4: 35-7.

3. Coles R, Kharasch ED. Stereoselective analysis of bupropion and hydroxybupropion in human plasma and urine by LC/MS/MS. J Chromatogr B Analyt Technol Biomed Life Sci 2007; 857: 67-75.

4. Balit CR, Lynch CN, Isbister GK. Bupropion poisoning: A case series. Med J Aust 2003; 178: 61-3.

5. Mercarolle M, Denooz R, Lachtre G, Charlier C. A fatal case of Bupropion (Zyban) overdose. J Anal Toxicol 2008; 32: 192-6.

6. Pesola GR, Avasarala J. Bupropion seizure proportion among new-onset generalized seizures and drug related seizures presenting to an emergency department. J Emerg Med 2002; 22: 235-9.

7. Druteika D, Zed PJ. Cardiotoxicity following bupropion overdose. Ann Pharmacotherapy 2002; 36: 1791-5.

8. Isbister GK, Buckley NA, Whyte IM. Serotonin toxicity: A practical approach to diagnosis and treatment. Med J Aust 2007; 187 (6): 361-5.

9. Jepsen F, Matthews J, Andrews F. Sustained release bupropion overdose: an important cause of prolonged symptoms after an overdose. Emerg Med J 2003; 20 (6): 560-1.

10. Caplan LR. Ptosis. J Neurol Neurosurg Psychiatry 1974; 37: 1-7.

11. Cartier L, Guzmán J, Pasquali R. Retracción palpebral de causa neurológica. Presentación de tres pacientes, interpretación patogénica. Rev Med Chile 2017; 145: 264-8.

12. Manconi M, Cesnik E, Casetta I, Govoni V, Ferini-Strambi L, Granieri E. Cerebral blepharoptosis: two new cases, literature review and proposal for diagnostic criteria. Neurol Sci 2006; 27: 161-5.

13. Chalela JA, Wolf RL, Maldjian JA, Kasner SE. MRI identification of early white matter injury in anoxic-ischemic encephalopathy. Neurology 2001; 56: 481-5.

14. Zamora CA, Nauen D, Hynecek R, Ilica AT, Izbudak I, Sair HI, et al. Delayed posthypoxic leukoencephalopathy: a case series and review of the literature. Brain Behav 2015; 5. Published online 015. doi: 10.1002/ brb3.364.

15. Howard RS, Holmes PA, Siddiqui A, Treacher D, Tsiropoulos I. Koutroumanidis M. Hypoxic-ischaemic brain injury: imaging and neurophysiology abnormalities related to outcome. Q J Med 2012; 105: 551-61. 